Check for updates

Cite this: J. Anal. At. Spectrom., 2021, 36,1272

Received 22nd December 2020 Accepted 6th May 2021

DOI: $10.1039 / \mathrm{d0ja00525h}$

rsc.li/jaas

\section{Introducing dimethyl carbonate as a new eluent in HPLC-ICPMS: stronger elution with less carbon $\uparrow$}

\begin{abstract}
Bassam Lajin (D) * and Walter Goessler (D)
Inductively coupled plasma mass spectrometry (ICPMS) offers unique possibilities as a chromatographic detector. A major limitation is the difficulty of employing conventional mobile phases in the reversedphase mode (i.e. containing significant amounts of organic solvents), without a modified instrumental setup that does not retain maximum sensitivity. We introduce dimethyl carbonate (DMC) as a new organic eluent in liquid chromatography with especially desirable properties that enable strong elution under standard ICPMS conditions. A total of eleven model compounds with various properties were tested and the elution behavior was compared with that for common organic eluents. At a concentration of $10 \% \mathrm{v} / \mathrm{v}$, a mobile phase flow rate of $0.3 \mathrm{~mL} \mathrm{~min}^{-1}$, and standard ICPMS conditions and set-up, DMC resulted in a stable plasma (reflected power $<5 \mathrm{~W}$ at a forward power of $1550 \mathrm{~W}$ ), no significant deposition of carbon on sampler and skimmer cones, and no change in sensitivity following at least 8 hours of continuous operation. At that concentration, DMC decreased the retention factors of compounds on a reversed-phase column by up to 40 -fold relative to $10 \%$ methanol. When averaged over all compounds tested, it was found that a $10 \% \mathrm{v} / \mathrm{v}$ concentration of DMC was equivalent to $23 \%$, and $48 \%$ of acetonitrile and methanol, respectively, none of which showed such compatibility with ICPMS at comparable conditions. Due to its unique chemical structure relative to common solvents, DMC was also found to confer different chromatographic selectivity. DMC yielded similar effects on sensitivity to methanol within the concentration range of $1.0-10 \% \mathrm{v} / \mathrm{v}$, with signal suppression/ enhancement factors within the range of 0.5-4.0 depending on the element. An application involving chlorine speciation in urine is additionally presented. In conclusion, the employment of DMC may facilitate the elution and detection of novel hydrophobic compounds and improve column recovery under standard ICPMS conditions and instrumental set-up without a compromise in detection limits.
\end{abstract}

\section{Introduction}

Inductively coupled plasma mass spectrometry (ICPMS) has been gaining increasing recognition as an element-selective detector in liquid chromatography. One of the most fascinating aspects of this technique when utilized as a chromatographic detector is that it not only enables quantitative speciation analysis which is normally solely dependent on the elemental content but also can serve as a discovery tool for element-tagged natural compounds. This aspect has been significantly extended with the relatively recent introduction of the triple quadrupole technology to ICPMS to include many naturally abundant elements with major polyatomic interferences such as the non-metals. ${ }^{\mathbf{1 - 4}}$

The most prominent disadvantage of ICPMS is the susceptibility of the signal to carbon and the instability of the plasma

Institute of Chemistry, Analytical Chemistry for Health and Environment, University of Graz, Austria.E-mail: bassam.lajin@uni-graz.at

$\dagger$ Electronic supplementary information (ESI) available. See DOI: 10.1039/d0ja00525h at high proportions of organic solvents. This limitation has a particularly severe impact on coupling reversed-phase liquid chromatography with ICPMS for speciation analysis. Nevertheless, incorporating high percentages of an organic solvent (generally $>10-20 \%$ depending on the conditions) using conventional chromatographic columns with conventional flow rates (0.1-1.0 $\mathrm{mL} \mathrm{min}^{-1}$ ) has been successfully practiced but necessitated approaches such as column flow splitting, postcolumn dilution, the employment of oxygen as an optional gas, and a modified instrumental set-up. ${ }^{\mathbf{1 - 4}}$ Apart from added complexity, these approaches can clearly compromise the detection limit.

Therefore, it is understandable that the majority of speciation studies by HPLC-ICPMS have employed little to no organic solvent in the mobile phase. It is not surprising however that many studies reported low column recoveries or, in other words, a "gap" between the total element content determined by ICPMS and the sum of individual chromatographic peaks detected. ${ }^{5-9}$ Undoubtedly, there would have been many more examples demonstrating the elegance of HPLC-ICPMS for the 
detection of novel element-tagged natural compounds with the employment of more suitable chromatographic conditions.

In an attempt to address this limitation, we recently introduced the concept of "ion-repulsion chromatography", which is merely a simple and often neglected aspect of ion-pair/ioninteraction chromatography, as an approach that was demonstrated to greatly facilitate the elution of charged compounds under $90-100 \%$ aqueous mobile phases. ${ }^{\mathbf{1 0}}$ Neutral nonionizable compounds would however not benefit from this approach, and the employment of an organic solvent for reversed-phase chromatography with an ICPMS detector remains inevitable.

The aim of the present work was to introduce dimethyl carbonate (DMC) as an organic solvent in liquid chromatography, to highlight the desirable properties that could render this solvent particularly useful for speciation analysis by HPLCICPMS, and to compare its performance with that of other commonly used solvents, particularly the most commonly employed methanol.

\section{Experimental}

The chromatographic experiments in the present work were undertaken using a reversed-phase column (Zorbax Eclipse Plus C18, $50 \mathrm{~mm} \times 2.1 \mathrm{~mm}$ i.d., $1.8 \mu \mathrm{m}$, Agilent Technologies,
Waldbronn, Germany). A short column with high efficiency was selected to facilitate the investigation and detection of strongly retained compounds with retention factors up to 100 under all chromatographic conditions to be tested. The vast majority of experiments were performed under the following chromatographic conditions: mobile phase flow rate: $0.3 \mathrm{~mL} \mathrm{~min}^{-1}$; column temperature: $40{ }^{\circ} \mathrm{C}$; injection volume: $1.0 \mu \mathrm{L}$; mobile phase: $5.0-20 \%$ of an organic solvent $+80-95 \%$ of an aqueous solution containing $50 \mathrm{mM}$ acetic acid with a $\mathrm{pH}$ buffered by adjustment with ammonia to 5.0. Deviations from these conditions are highlighted in the respective figure caption. DMC-containing mobile phases were thoroughly mixed and sonicated for $10 \mathrm{~min}$.

To ensure a thorough chromatographic investigation, care was taken to select a group of candidate analytes with varied properties in terms of hydrophobicity, ionizability, and polarity (Fig. 1). Since not all of the compounds available to us were amenable to ICPMS detection and in order to avoid bias towards certain classes of compounds that are detectable by ICPMS, a few compounds such as caffeine, theobromine, aspirin, and acetophenone were included in the study and the chromatographic behavior for these was investigated with an Agilent 1260 diode array spectrophotometric detector coupled with an Agilent 1260 Infinity II chromatographic system (Agilent Technologies, Waldbronn, Germany) while employing identical<smiles>NS(=O)(=O)c1ccccc1</smiles>

Benzenesulfonamide<smiles>O=C(O)Cc1ccccc1Nc1c(Cl)cccc1Cl</smiles>

Diclofenac<smiles>O=C(O)C(Br)(Br)Br</smiles>

Tribromoacetic acid<smiles>Cc1ccc(S(N)(=O)=O)cc1</smiles>

Toluenesulfonamide<smiles>Cn1c(=O)c2c(ncn2C)n(C)c1=O</smiles>

Caffeine<smiles>Cn1cnc2c1c(=O)[nH]c(=O)n2C</smiles>

Theobromine<smiles>O=C(O)c1ccccc1SSc1ccccc1C(=O)O</smiles>

Dithiodibenzoic acid<smiles>O=c1n(CCCN2CCN(c3cccc(Cl)c3)CC2)nc2ccccn12</smiles>

Trazodone<smiles>CC(=O)c1ccccc1</smiles>

Acetophenone<smiles>CCCCCCS(=O)(=O)O</smiles>

Hexanesulfonic acid<smiles>CC(=O)Oc1ccccc1C(=O)O</smiles>

Aspirine

Fig. 1 Chemical structures of the compounds included in the study. To provide wider options in terms of including compounds with different chemical structures for a detailed chromatographic investigation, some compounds not amenable to detection by ICPMS were included and the chromatographic behavior of these were investigated with HPLC-UV. Information about the hydrophobicity and ionization of the compounds can be found in Table 1. 
chromatographic conditions to those above mentioned. The concentration of injected compounds $(1.0 \mu \mathrm{L})$ was $1.0-20 \mathrm{mg}$ element $\mathrm{L}^{-1}$ for ICPMS detection and 50-100 mg compound $\mathrm{L}^{-1}$ for UV detection. All compounds were prepared in pure water.

ICPMS detection was performed using an Agilent 8900 ICPQQQ (inductively coupled plasma triple quadrupole mass spectrometry, ICPMS/MS) system coupled with an Agilent 1100 HPLC system. A standard ICPMS/MS set-up was employed for all experiments, consisting of an AriMist polyether ether ketone (PEEK®) nebulizer (maximum nebulizer gas flow rate 0.8 $\mathrm{L} \min ^{-1}$ ), a glass double pass spray chamber, nickel/copper sampler and skimmer cones, and a quartz plasma torch with an inner diameter of $2.5 \mathrm{~mm}$. Using oxygen as an optional gas was avoided in all experiments.

The operating ICPMS/MS parameters were as follows unless otherwise stated: RF (radiofrequency) power: $1550 \mathrm{~W}$; Plasma gas: 15.0 L min ${ }^{-1}$; Auxiliary gas: $0.9 \mathrm{~L} \mathrm{~min}^{-1}$; RF matching: 1.80; sampling position (sampling depth): $6.0 \mathrm{~mm}$; nebulizer gas flow rate: $0.65 \mathrm{~L} \mathrm{~min}^{-1}$; makeup gas (argon) flow rate: $0.30 \mathrm{~L} \mathrm{~min}^{-1}$; optional gas: $0.0 \%$; nebulizer pump speed (for drainage): 0.50 rps (ca. $2.0 \mathrm{~mL} \mathrm{~min}^{-1}$ ); S/C (spray chamber) temp.: $2{ }^{\circ} \mathrm{C}$. Chlorine and bromine were detected in the hydrogen mode $\left(\mathrm{H}_{2}\right.$ flow rate: $3.5 \mathrm{~mL} \mathrm{~min}^{-1}$ ) by monitoring the mass transitions $35 \rightarrow 37$ and $79 \rightarrow 80$, respectively. Sulfur was detected in the oxygen

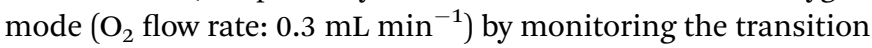
$32 \rightarrow 48$.

All reagents included in the study were of HPLC grade, where available, with a purity of $\geq 99 \%$. Dimethyl carbonate was purchased from Sigma-Aldrich (ReagentPlus ${ }^{\circledR}$, purity $\geq 99 \%$, Sigma-Aldrich, Steinheim, Germany). Purified water (18.2 $\mathrm{M} \Omega \mathrm{cm})$ was produced in-house using a Milli-Q water purification system (Millipore GmbH, Vienna, Austria).

\section{Results and discussion}

The rationale behind our selection of dimethyl carbonate was based on fulfilling the following criteria: (1) high hydrophobicity which generally correlates with elution strength and necessitates lower organic content and carbon load, (2) an oxygen to carbon ratio of at least 1.0 which in our experience confers better tolerability by ICPMS, and (3) high boiling point and low vapor pressure to decrease the carbon load. Compared with the most commonly used solvents acetonitrile and methanol, dimethyl carbonate shows superior properties in terms of the above criteria, with a much higher $\log P$ of 0.5 , a boiling point of $91{ }^{\circ} \mathrm{C}$, and vapor pressure of $55 \mathrm{mmHg}$ (at $25{ }^{\circ} \mathrm{C}$ ) (for more details see supplementary Table $\mathrm{S} 1 \dagger)$. While this is the first time dimethyl carbonate is used as an eluent in liquid chromatography in general, the use of a structurally related compound, propylene carbonate, has been previously described in liquid chromatography, ${ }^{\mathbf{1 1 - 1 3}}$ but the potential of no member in this class of organic solvents has been investigated in combination with ICPMS when used as an element-selective chromatographic detector. However, we encountered a report by Wei et al., ${ }^{\mathbf{1 4}}$ describing the coupling of a single cell sampling system with ICPMS, where it was mentioned that due to its high oxygen content dimethyl carbonate was employed as an "oxygen compensation reagent", acting as an oxygen source in order to aid in the combustion of carbon resulting from the introduction of a hexanol phase. ${ }^{\mathbf{1 4}}$

Unlike acetonitrile, methanol confers much higher plasma stability and tolerability by ICPMS, and, depending on the conditions (e.g. flow rate), up to $c a .25 \% \mathrm{v} / \mathrm{v}$ of methanol can be tolerated under standard ICPMS set-up and without the need for oxygen as an optional gas. At concentrations within the range of $5.0-10 \%$, dimethyl carbonate showed astonishingly higher elution strength compared to methanol at equal concentrations, decreasing the retention factor for some compounds by up to 38 -fold (Fig. 2). The effects of dimethyl carbonate were found to be relatively dependent on the compound tested and therefore a change in chromatographic selectivity and a clear shift in peak order was observed relative to methanol at comparable concentrations (Fig. 2).

It was essential to rule out artefacts contributing to the displayed elution behavior of dimethyl carbonate compared with methanol. To ensure that the chromatographic separation is undertaken predominantly under a reversed-phase mode, we tested the retention of doubly charged anions and cations under $10 \%$ dimethyl carbonate in comparison with $10 \%$ methanol. No retention was observed for calcium and sulfate, ruling out the possibility of the presence of acids/bases as impurities in dimethyl carbonate which would display ion-pairing/ionrepulsion behavior (Fig. S1†). Dimethyl carbonate has been previously described as a green carbonylating and alkylating reagent. ${ }^{15}$ Even though the reactivity of dimethyl carbonate is relevant under conditions far from what is normally used in liquid chromatography (e.g. $\geq 200{ }^{\circ} \mathrm{C}$, extreme $\mathrm{pH}$ values, etc. $){ }^{15}$ we ensured that the chemical structures of some of the tested compounds, which showed a dramatic change in retention behavior, remained unaltered by monitoring the signal with a molecule selective detector (electrospray ionization mass spectrometry, ESI-MS) (Fig. S1†).

While the high hydrophobicity can explain the strong elution of dimethyl carbonate on the $\mathrm{C} 18$ reversed-phase column as indicated by the $\log P$ value (ESI Table S1 $\dagger$ ), the origin of the observed shift in selectivity for some of the compounds is not entirely clear. We note however that unlike other commonly used eluents, dimethyl carbonate has a high hydrogen bond acceptance count (3) and no capacity as a hydrogen bond donor (ESI Table S1 $\dagger$ ). This significant imbalance between hydrogen bond acceptance and donation capacity likely exerts a significant impact on selectivity.

The pattern of a clearly stronger elution than methanol was consistent in all compounds tested (Table 1). It is noteworthy however that dimethyl carbonate was observed to yield increased peak tailing relative to methanol (Table 1). The tailing behavior was found to be dependent on the conditions (e.g. adjusting $\mathrm{pH}$ decreased the asymmetry factor (As, calculated as the ratio $b / a$ (tail width/front width) at $10 \%$ peak height) for the ionizable compound trazodone from 2.4 to 1.2), and on the other hand, methanol resulted in similar peak tailing behavior to DMC for caffeine when used at concentrations that promote comparably fast elution (ESI Fig. S2 $\dagger$ ). 


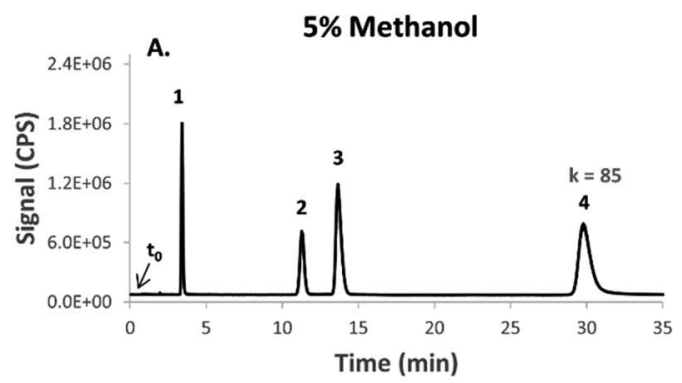

\section{5\% Dimethylcarbonate}

c.

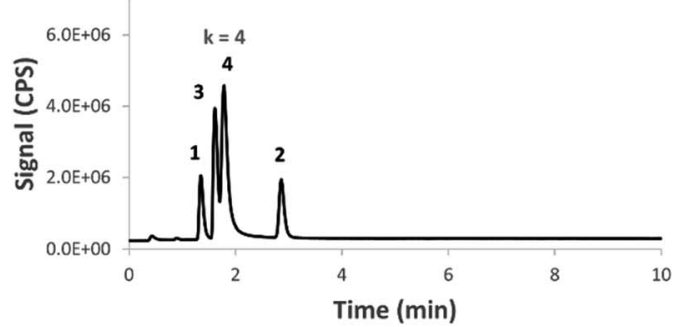

$10 \%$ Methanol

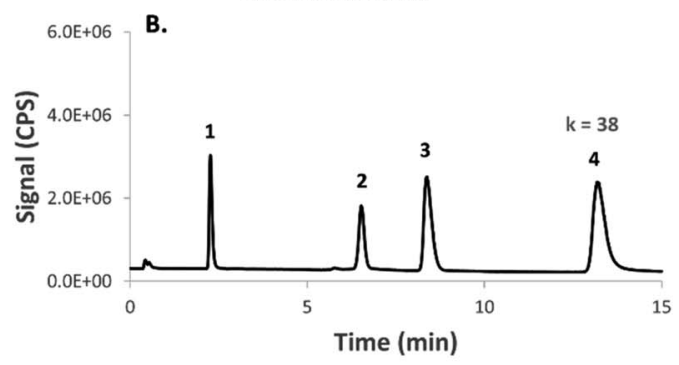

10\% Dimethylcarbonate

D.

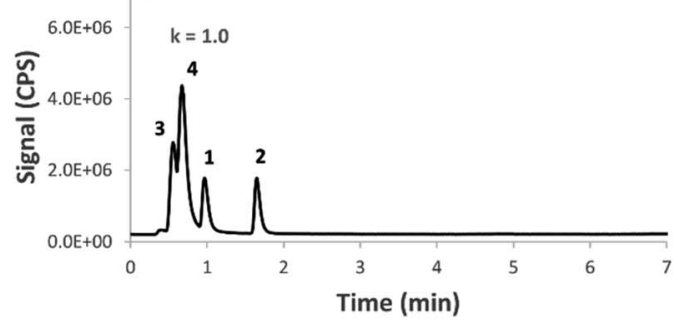

Fig. 2 A comparison of the elution strength and chromatographic selectivity between dimethyl carbonate (DMC) and methanol. The chromatographic conditions were identical (see Experimental) except for incorporating the indicated concentration of organic solvent (in v/v\%). Note the change in selectivity and the high elution strength for DMC relative to methanol, particularly for some of the compounds (peak 3 and peak 4). The retention factor for peak 4 is indicated on the chromatograms as an example to quantitatively highlight this observation (column void time $t_{0}$ $=0.35 \mathrm{~min}$ ). For an overview over all of the tested compounds see Table 1. Benzenesulfonamide (1); toluenesulfonamide (2); hexane sulfonic acid (3); dithiodibenzoic acid (4).

Table 1 Key properties and chromatographic behavior of tested compounds under buffered aqueous mobile phases containing $10 \%$ dimethyl carbonate (DMC) or $10 \%$ methanol

\begin{tabular}{|c|c|c|c|c|c|c|}
\hline & \multirow[b]{2}{*}{$\log P^{a}$} & \multirow[b]{2}{*}{ Charge state $^{b}$} & \multicolumn{2}{|l|}{ Retention factor } & \multicolumn{2}{|c|}{ Asymmetry factor $^{e}$} \\
\hline & & & $10 \%$ methanol & $10 \% \mathrm{DMC}$ & $\begin{array}{l}10 \% \\
\text { methanol }\end{array}$ & $10 \% \mathrm{DMC}$ \\
\hline Theobromine & -0.8 & 0 & 3.3 & 0.21 & 2.0 & 2.0 \\
\hline Caffeine & -0.1 & 0 & 14 & 0.54 & $1.3^{c}$ & 2.3 \\
\hline Aspirin & 1.2 & -1 & 3.6 & 0.31 & 1.9 & 2.3 \\
\hline Acetophenone & 1.6 & 0 & 40 & 14 & 1 & 1 \\
\hline Diclofenac & 4.4 & -1 & $>100^{d}$ & 13 & NA & 1.6 \\
\hline Trazodone & 2.8 & +1 & $>100$ & 11 & NA & $2.4^{c}$ \\
\hline Hexanesulfonic acid & 1.4 & -1 & 23 & 0.6 & 1.6 & 1.9 \\
\hline Tribromoacetic acid & 2.2 & -1 & 3.8 & 0.37 & 2 & 2.5 \\
\hline Benzenesulfonamide & 0.3 & 0 & 5.6 & 1.9 & 1.5 & 2.1 \\
\hline Toluenesulfonamide & 0.8 & 0 & 18 & 3.8 & 1.1 & 2.0 \\
\hline Dithiodibenzoic acid & 3.1 & -2 & 38 & 1 & 1.9 & 2.1 \\
\hline
\end{tabular}

${ }^{a}$ Computed by XLogP3 3.0 (PubChem release 2019.06.18). ${ }^{b}$ Calculated based on the employed mobile phase pH $50 \mathrm{mM}$ ammonium acetate 5.0 for all analytes except for diclofenac where $50 \mathrm{mM}$ ammonium bicarbonate $\mathrm{pH} 7.0$ was used due to low water solubility at acidic $\mathrm{pH} .{ }^{c}$ The asymmetry factor was found to be dependent on the conditions. At $30 \%$ methanol the asymmetry was 2.3 for caffeine. At pH 6.0 , the asymmetry factor for trazodone was 1.2 (see also ESI Fig. S2). ${ }^{d}$ No peak was observed for diclofenac under $10 \%$ methanol within a chromatographic runtime corresponding to a retention factor of $100 .{ }^{e}$ Tailing was assessed by the asymmetry factor calculated as the ratio $b / a$ (tail width/front width) at $10 \%$ peak height.

Compared with other solvents, dimethyl carbonate offered clearly stronger elution strength than acetonitrile for all tested compounds and stronger elution strength than isopropanol for most compounds (Fig. 3 and ESI Fig. S3†). Overall, calculations based on the linear regression lines (average $r^{2}=0.999$ ) depicting the logarithmic relationship between the retention factor and the percentage concentration of the organic eluent ( $\log k v s . \log C \%)$, revealed that the concentrations equivalent in elution strength to $10 \%$ dimethyl carbonate, when averaged on the different compounds, amounted to $13 \%, 23 \%$, and $48 \%$ for isopropanol, acetonitrile, and methanol, respectively (Fig. 4). These calculations were validated in practice for a few 
Theobromine

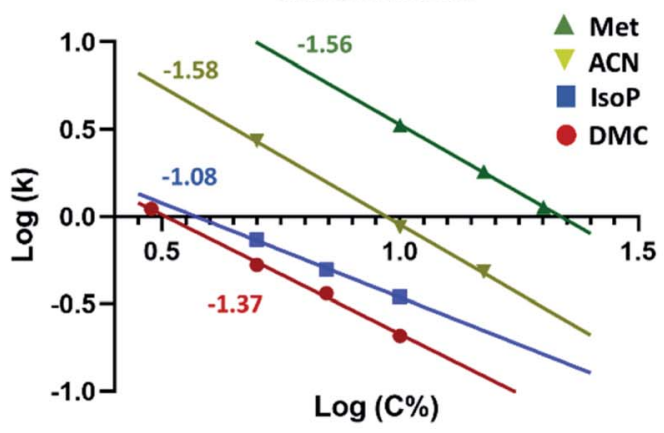

Dithiodibenzoic acid

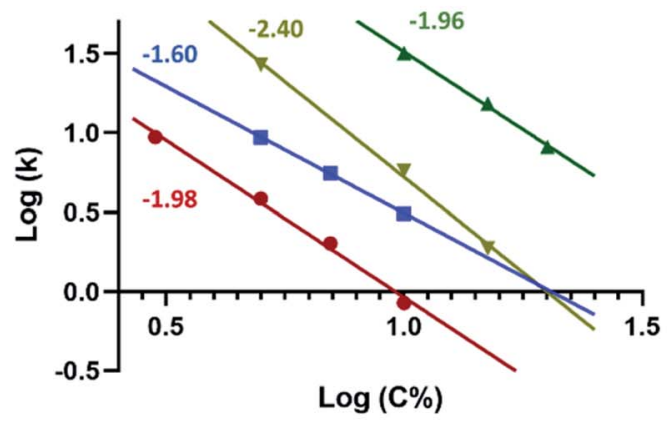

Aspirin

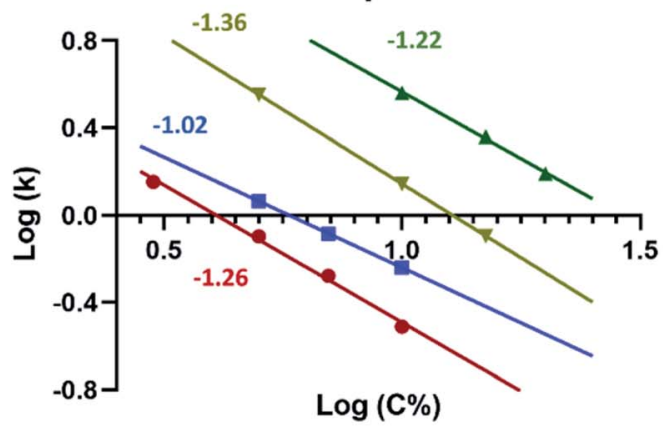

Caffeine

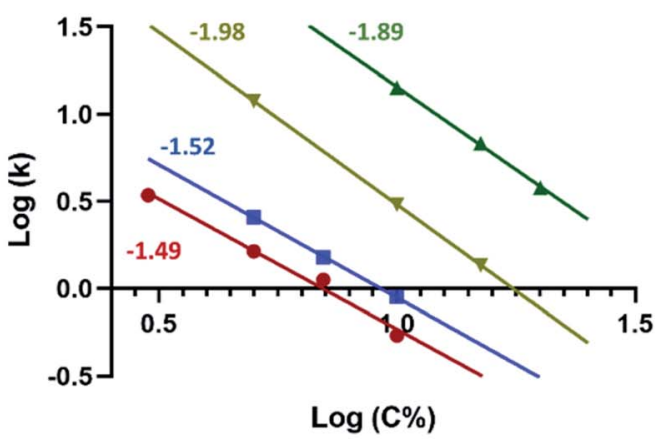

Acetophenone

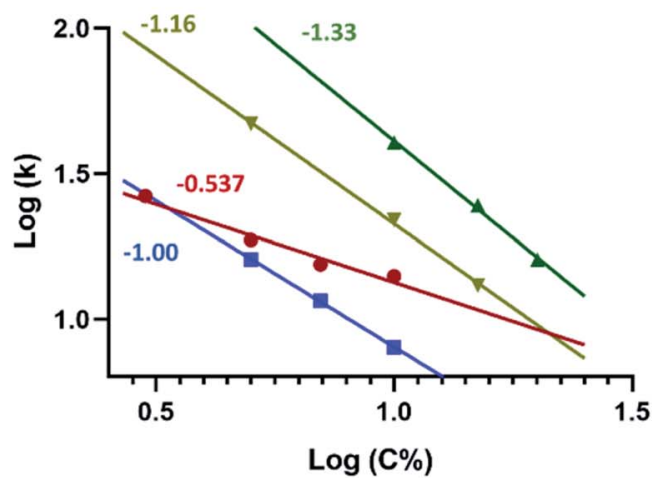

Toluenesulfonamide

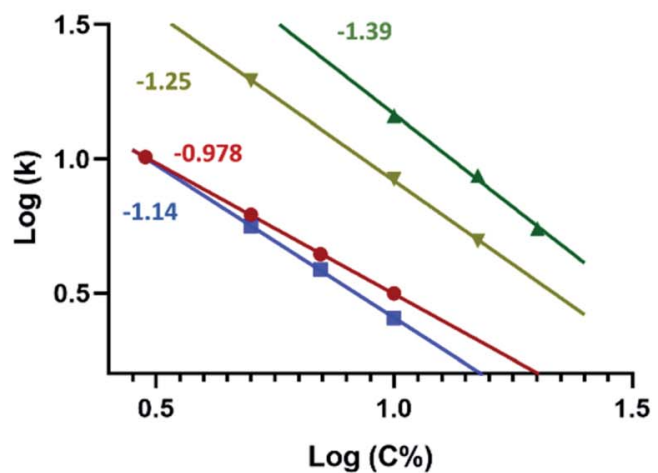

Fig. 3 A comparison of the concentration dependent elution behavior between dimethyl carbonate (DMC) and some of the most commonly employed organic solvents in liquid chromatography, namely, methanol (Met), acetonitrile (ACN), and isopropanol (IsoP). The plots show the relationship between the logarithmic retention factor and organic solvent concentration (in v/v\%). Compounds included in this investigation were: theobromine, caffeine, dithiodibenzoic acid, acetophenone, aspirin, and toluenesulfonamide. The numbers indicate the slopes of the regression lines. Note the wider discrepancy in the slopes for certain pairs of compounds (e.g. dithiodibenzoic acid and toluene sulfonamide) under certain solvents (e.g. DMC vs. methanol), indicating different impacts on selectivity.

compounds (e.g. see ESI Fig. S2B and $\left.\mathrm{C}^{\dagger}\right)$. Under the standard experimental conditions employed in the present work (see Experimental), the plasma was unstable for the above equivalent concentrations of methanol and acetonitrile. Isopropanol on the other hand was well tolerated at 10-15\%. Compared to $10 \%$ dimethyl carbonate, a concentration of $15 \%$ isopropanol yielded slightly increased carbon build-up on the sampler and skimmer cones (ESI Fig. S5 $\dagger$ ) after 8 hours of operation but, as with dimethyl carbonate, no significant change in sensitivity was observed. The main disadvantage with isopropanol (as with water/methanol mixtures, particularly at ratios close to 1.0) is however the markedly increased column back pressure due to its significantly higher viscosity of 2.0 compared with $0.67 \mathrm{mPa}$ at $20{ }^{\circ} \mathrm{C}$ for dimethyl carbonate (for more information see supplementary Table S1†). High viscosity also compromises chromatographic efficiency as is clearly indicated by the peak widths in Fig. S2B and C. $\dagger$ 


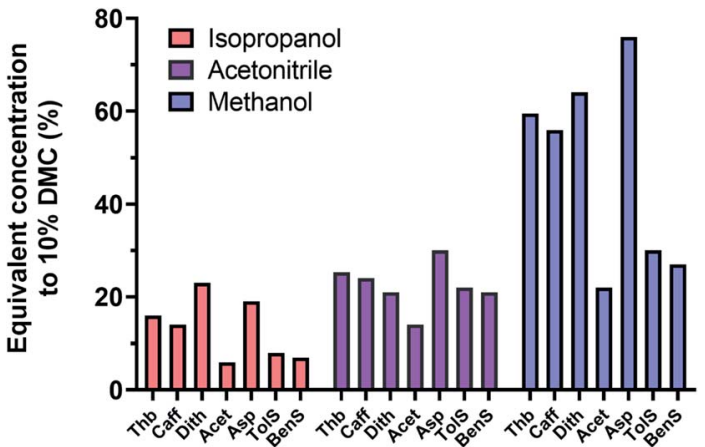

Fig. 4 Calculated concentrations of the investigated organic solvents expected to yield similar elution strength to $10 \%$ DMC. The calculations were based on the regression lines obtained in the investigation illustrated in Fig. 3. Some of the calculations were validated experimentally e.g. the calculated equivalent concentration of methanol for $5 \%$ DMC was $31 \%$ and the experimentally determined value was ca. $30 \%$ (ESI Fig. S2A and $\mathrm{B} \dagger$ ). On average, the 10\% DMC equivalent concentrations of methanol, acetonitrile, and isopropanol were $14 \%$, $23 \%$, and $48 \%$, respectively.

The impact of dimethyl carbonate on ICPMS sensitivity was investigated after normalization to the signal under a $100 \%$ aqueous mobile phase and compared with methanol at identical concentrations and experimental conditions (Fig. 5). The signal suppression/enhancement effects observed for the variety of elements tested were as predicted and comparable to methanol, with a mild shift in the peak behavior concentrations due to the different molar concentration of carbon in methanol (25 M) compared with dimethyl carbonate (35 M) (Fig. 5). It is noteworthy that the observed signal suppression/enhancement factors do not only reflect carbon effects but also slight differences in nebulization efficiency. Indeed, the maximum sensitivity observed for each eluent composition was achieved using a slightly different carrier gas flow rate, with higher organic proportions necessitating a lower carrier gas flow rate (particularly for dimethyl carbonate), but the optimum nebulizer gas flow rate for all experiments (including 100\% aqueous) was always within the range of $0.9-1.1 \mathrm{~L} \mathrm{~min}^{-1}$. Operating at the optimum carrier gas flow rates for a $100 \%$ aqueous mobile phase ( $c a$. 1.05 $\mathrm{L} \mathrm{min}^{-1}$ ) when using $10 \%$ dimethyl carbonate (optimum flow: $0.9 \mathrm{~L} \mathrm{~min}^{-1}$ ) was observed to result in a two-fold sensitivity loss. It is therefore recommended to optimize this setting for maximum sensitivity when using dimethyl carbonate.

As an application, the chlorine metabolome in two morning urine samples collected from one volunteer taking chlorinecontaining medication was examined and the peak patterns were compared using different concentrations of methanol and dimethyl carbonate (Fig. 6). A larger number of "hits" were detected under dimethyl carbonate (at 5\%, Fig. 6C and D, and at $10 \%$, Fig. $6 \mathrm{E}$ and $\mathrm{F}$ ) than under $20 \%$ methanol (Fig. $6 \mathrm{~A}$ and $\mathrm{B}$ ). Overall, the sum of detected chlorine peaks under $20 \%$ methanol, 5\% DMC, and 10\% DMC were ca. 7.5, 8.5, and $10 \mathrm{mg} \mathrm{Cl}$ per L, respectively which yields an improvement by $14 \%$ and $33 \%$ under 5\% DMC and 10\% DMC, respectively, compared
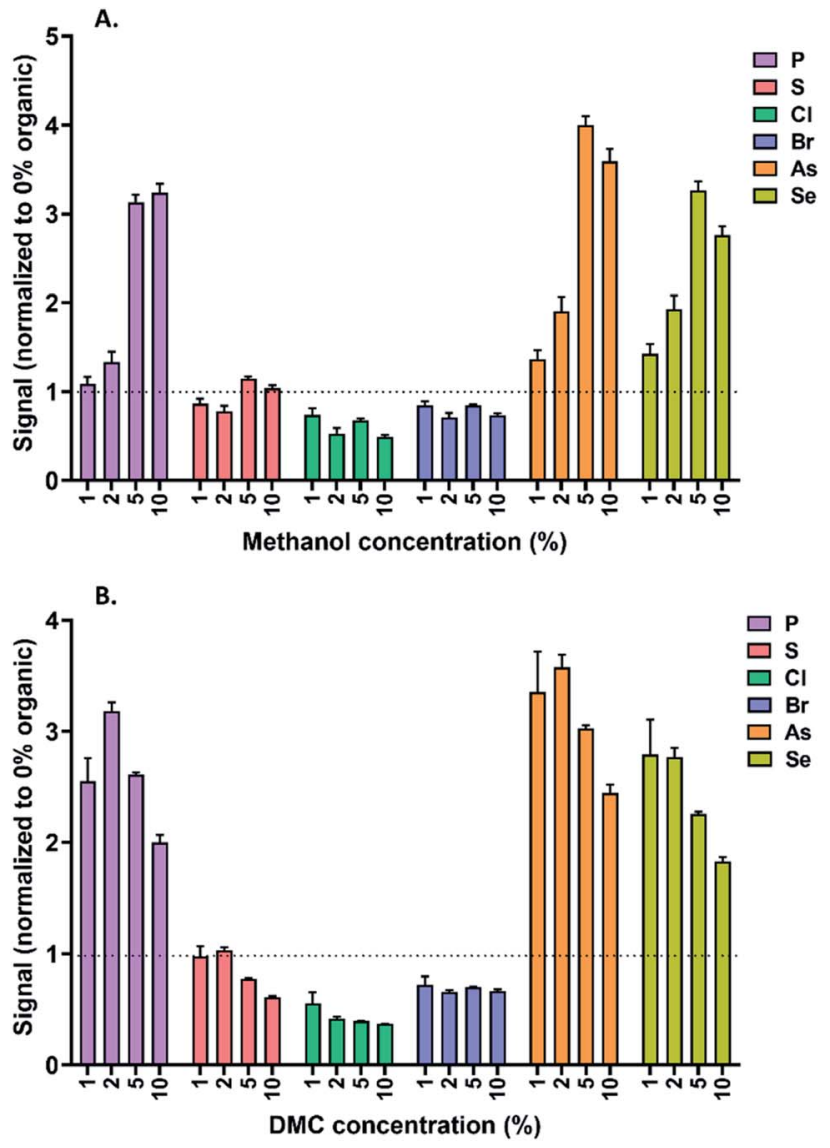

Fig. 5 Sensitivity of ICPMS detection for a range of element under different compositions of carrier solution (or mobile phase). The graphs illustrate the signal normalized to $100 \%$ aqueous (containing $50 \mathrm{mM}$ ammonium acetate buffer $\mathrm{pH}$ 5.0). The experiments were performed under identical conditions to those used in chromatography except for using a stainless steel restrictive capillary $(5 \mathrm{~m})$ in place of the chromatographic column to save time. A mixture containing $1 \mathrm{mg} \mathrm{L}^{-1}$ of each element in inorganic form was injected $(1 \mu \mathrm{L})$. Note that the signal factors reflect not only the carbon enhancement/ suppression effects relative to $100 \%$ aqueous but also the nebulization efficiency which is a rate-limiting factor in ICPMS detection and is dependent on the composition of the carrier solution/mobile phase. Therefore, and while all instrumental conditions were identical, it was necessary to adjust the carrier gas flow for each carrier solution composition in order to maximize the accuracy of the comparisons. Lower flow rates were optimal for higher organic solvent contents, particularly for DMC, but the applied values were always in the range of $0.9-1.1 \mathrm{~L} \mathrm{~min}$.

$20 \%$ methanol. Higher concentrations of methanol than $20 \% \mathrm{v} /$ $\mathrm{v}$ were not well-tolerated by ICPMS under the standard conditions employed.

Fig. $6 \mathrm{E}$ and $\mathrm{F}$ also show the chlorine profile in the two investigated urine samples after spiking with the chlorine containing drug diclofenac with a mobile phase containing $10 \%$ DMC. A mobile phase containing $48 \%$ methanol (with UV detection) was needed to achieve a comparable retention time to that under $10 \%$ DMC.

Dimethyl carbonate is an ester and can be subject to hydrolysis. We investigated this aspect by monitoring the retention stability at 
A. $S 1-20 \%$ Methanol

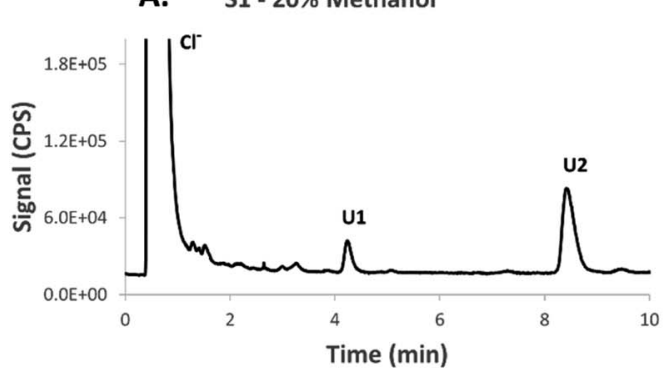

C. $\quad \mathrm{S} 1-5 \% \mathrm{DMC}$

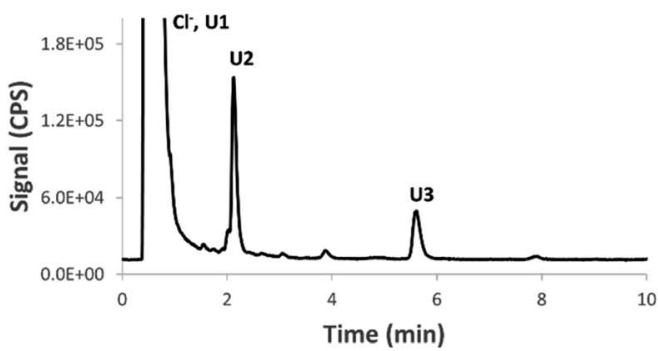

E. $\quad \mathrm{S1}-10 \% \mathrm{DMC}$

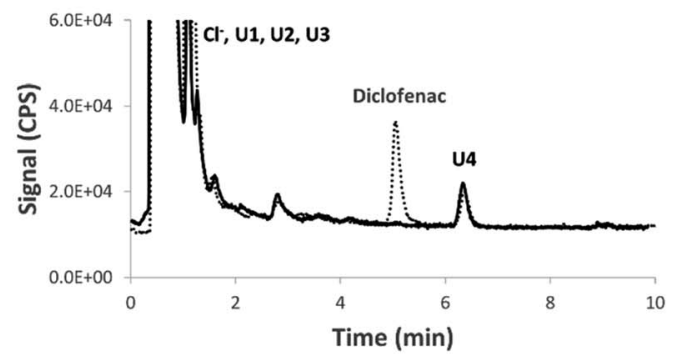

B. $\mathrm{S} 2-20 \%$ Methanol

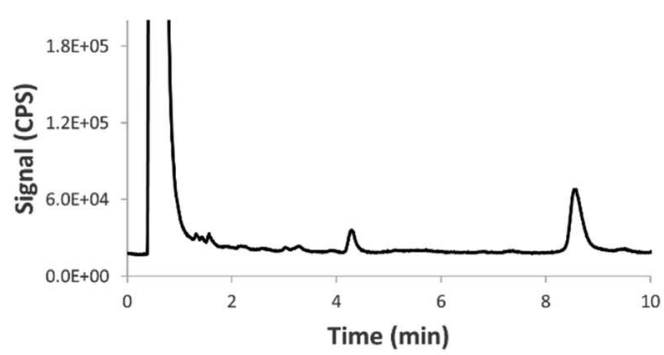

D. $\mathrm{S2}-5 \% \mathrm{DMC}$

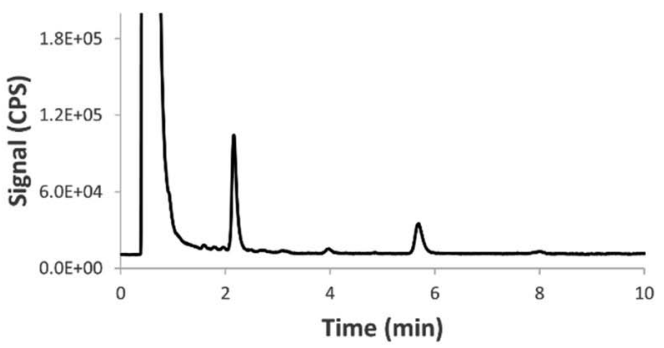

F.

S2 - 10\% DMC

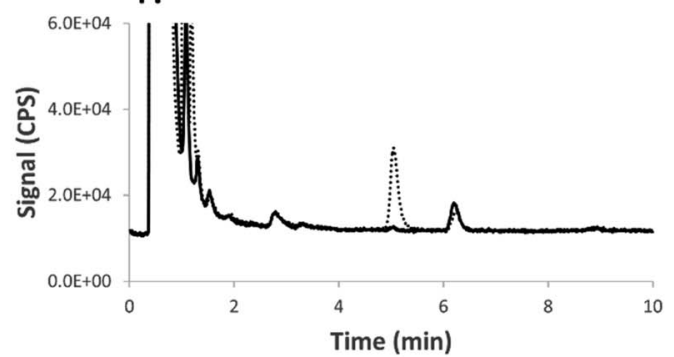

Fig. 6 The application of dimethyl carbonate as an eluent for chlorine speciation in urine by HPLC-ICPMS/MS. The chromatograms show the detection of chlorine species in two different morning urine samples from one volunteer. The urine samples shown in Fig. $6 \mathrm{E}$ and were spiked with $1.0 \mathrm{mg} \mathrm{Cl}$ per $\mathrm{L}$ of the chlorine containing drug diclofenac ( $\mathrm{OOD}=30 \mu \mathrm{g} \mathrm{Cl}$ per L, injection volume: $5.0 \mu \mathrm{L}$ ).

various buffered mobile phase $\mathrm{pH}$ values. No significant change in retention was observed over three hours of measurement time (ESI Fig. S4 $\dagger$ ). Furthermore, a 10\% dimethyl carbonate-containing mobile phase $(\mathrm{pH} 5.0)$ showed a retention time of $1.65 \pm$ $0.01 \min (k=3.7)$ for toluene sulfonamide and a retention time of $1.63 \pm 0.01 \mathrm{~min}$ following storage at room temperature for 5 days in a closed glass container.

The main disadvantage of dimethyl carbonate, especially as a general eluent for reversed-phase liquid chromatography, is its low water miscibility $(13 \% \mathrm{v} / \mathrm{v})$. Therefore, the maximum practical concentration of dimethyl carbonate is $10 \% \mathrm{v} / \mathrm{v}$. This concentration was however sufficient to alter chromatographic selectivity and elution profile and may serve as solvent additive in liquid chromatography in general. The UV-cutoff for pure dimethyl carbonate was found to be $220 \mathrm{~nm}$ (Fig. S6†) and that for a 10\% aqueous solution <200 nm. Furthermore, while we did not observe any plasma instability at the optimum flow rates for $2.1 \mathrm{~mm}$ i.d. columns $\left(0.3 \mathrm{~mL} \mathrm{~min}^{-1}\right)$, as indicated by a stable reflected power of $<5 \mathrm{~W}$ under an RF matching setting at $1.8 \mathrm{~V}$, a rapid increase in reflected power and plasma instability were observed under flow rates $>0.4 \mathrm{~mL} \mathrm{~min}^{-1}$ under the standard conditions employed. As the aim of the study was to introduce alternative solutions compatible with standard conditions and instrumental set-up, improvement of stability at higher flow rates by employing the "organic ICPMS mode" (1.5 mm plasma torch, oxygen as an option gas, sub-zero spray chamber temperature etc.) was not investigated.

\section{Conclusion}

Dimethyl carbonate is a new eluent in liquid chromatography. It

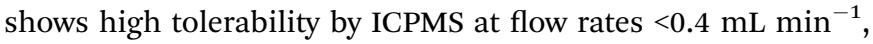
remarkably strong elution for hydrophobic compounds at low carbon loads, can be utilized to modify chromatographic selectivity, and is compatible with $2.1 \mathrm{~mm}$ i.d. chromatographic columns at their optimum flow rates $\left(0.2-0.4 \mathrm{~mL} \mathrm{~min}^{-1}\right)$ under standard ICPMS conditions and instrumental set-up.

\section{Author contributions}

Bassam Lajin: conceptualization, methodology, validation, investigation, writing - original draft, visualization. Walter 
Goessler: writing - review \& editing, project administration, funding acquisition.

\section{Conflicts of interest}

There are no conflicts to declare.

\section{References}

1 R. A. Glabonjat, G. Raber, K. B. Jensen, J. Ehgartner and K. A. Francesconi, Anal. Chem., 2014, 86(20), 10282-10287.

2 N. L. A. Jamari, J. F. Dohmann, A. Raab, E. M. Krupp and J. Feldmann, Anal. Chim. Acta, 2019, 1053, 22-31.

3 B. Klencsár, L. Balcaen, F. Cuyckens, F. Lynen and F. Vanhaecke, Anal. Chim. Acta, 2017, 974, 43-53.

4 M. J. Ruiz-Chancho, M. S. Taleshi, W. Goessler and K. A. Francesconi, J. Anal. At. Spectrom., 2012, 27(3), 501-504.

5 T. Jäger, H. Drexler and T. Göen, J. Anal. At. Spectrom., 2013, 28(9), 1402-1409.

6 D. Kuehnelt, N. Kienzl, P. Traar, N. H. Le, K. A. Francesconi and T. Ochi, Anal. Bioanal. Chem., 2005, 383(2), 235-246.
7 B. Lajin and W. Goessler, Anal. Chim. Acta, 2019, 1092, 1-8. 8 A. Pell, G. Kokkinis, P. Malea, S. A. Pergantis, R. Rubio and J. F. López-Sánchez, Chemosphere, 2013, 93(9), 2187-2194.

9 R. Schaeffer, K. A. Francesconi, N. Kienzl, C. Soeroes, P. Fodor, L. Váradi, R. Raml, W. Goessler and D. Kuehnelt, Talanta, 2006, 69(4), 856-865.

10 B. Lajin and W. Goessler, J. Chromatogr. A, 2020, 1631, 461575.

11 F. Tache, S. Udrescu, F. Albu, F. Micăle and A. Medvedovici, J. Pharm. Biomed. Anal., 2013, 75, 230-238.

12 N. Varsha, V. Pratibha, M. Soni, B. Ashok and B. Suvarna, J. Liq. Chromatogr. Relat. Technol., 2014, 37(8), 1094-1103.

13 N. Varsha, B. Suvarna, V. Pratibha, M. Soni and B. Ashok, J. Liq. Chromatogr. Relat. Technol., 2012, 35(18), 2643-2654.

14 X. Wei, D.-H. Zheng, Y. Cai, R. Jiang, M.-L. Chen, T. Yang, Z.-R. Xu, Y.-L. Yu and J.-H. Wang, Anal. Chem., 2018, 90(24), 14543-14550.

15 P. Tundo, M. Musolino and F. Aricò, Green Chem., 2018, 20(1), 28-85. 